Case Report

Open Access

CrossMark

\title{
Revascularization in multiple vessels
}

\begin{abstract}
It is male patient aged 60 with a history of Chronic hypertension, dyslipidemia, and Chronic smoker. 5years ago the patient had an aorto coronary bypass to present box angina class III of Canadian society of cardiology. 6months after surgery for coronary Revascularization presented symptoms of progressive angina to be why undergoes a percutaneous coronary intervention (PCI) of native vessels in 2times again class III (SCC), achieving complete Revascularization after placement of medicated sten (endeavor, Medtronic) zotarolimuseluting. With excellent angiographic and Clinical outcome. The patient remains asymptomatic and makes normal life.
\end{abstract}

Volume 3 Issue 5 - 2015

\section{Hugo Chinchilla Calix}

Chief of Cardiology, USA

Correspondence: Hugo Chinchilla, Chief of Cardiology, Military Hospital, Tegucigalpa, Honduras, El Ocotal, Francisco Morazan, USA, Email hugo_chinchilla@hotmail.com

Received:December 13, 2014 | Published: October 12, 2015

Keywords: coronary artery bypass grafting; percutaneous coronary intervention; class iii (nv) native vessels; drug-eluting sten

\section{History}

The patient is a 60year old man with a known history of Chronic hypertension controlled with enalapril $20 \mathrm{mg}$ per day and mixed uncontrolled dyslipemia. He doesnot have previous history of smoking or diabetes. Five years ago he under went cardiac bypass surgery that was complicated immediately with mediastin it is secondary to a wound infection over the sternum; for this reason he was admitted to the intensive care unit where he remained for two months. Six months after being discharged, he presented with anginal Chest pain with dyspnea that rapidlyprogressed to minimaleffortseven with intensive medical support. Wemethimfor the first time onNovember of 2007 when he had a positive stress test early (positive at 1minute 10seconds) in stage I of the BRUCE protocol with ST depressionanteriorly and inferiorly with a delayedrecoveryclinically and electrocardiographically.

\section{Angiography}

a. Normal LM.

b. Severe angled lesion at the level of the proximal third of the left anterior descending artery.

c. Critical lesion at the level of ramus intermedius rami.

d. Severe lesion at the level of the distal third of the CX v OM.

e. Right coronary artery with total occlusion at the proximal third (Figure 1-3).

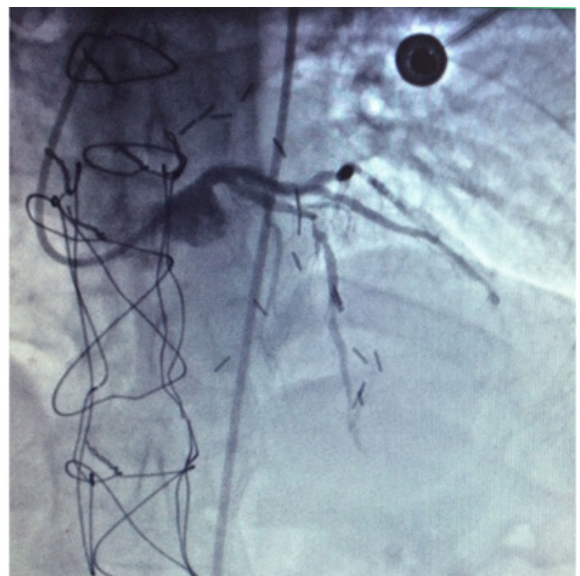

Figure I Right coronary artery with total occlusion at the proximal third.

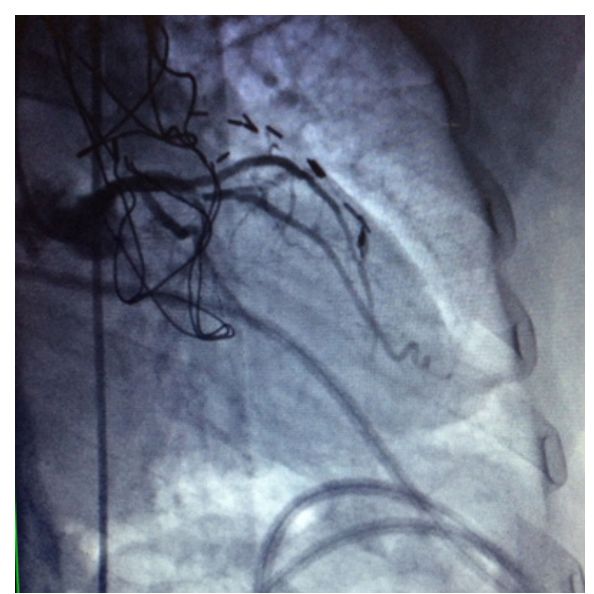

Figure 2 Right coronary artery with total occlusion at the proximal third.

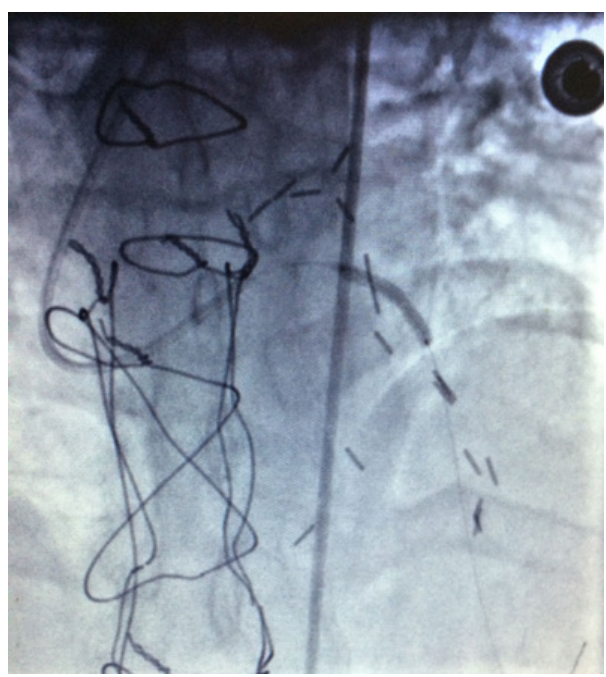

Figure 3 Through a conventional right femoral approach, the left coronary ostium was canalized using a $4.06 \mathrm{Fr}$ Launcher guiding catheter (Medtronic). Subsequently, a floppy microguide at the level of the anterior left descending artery was used to cross the lesion. Balloon dilation was performed to open the vessel starting with a $1.5 \times 18 \mathrm{~mm}$ Sprinter balloon (Medtronic) followed by a $2.0 \times 22 \mathrm{~mm}$ to finally deploy a $2.5 \times 18 \mathrm{~mm}$ Endeavor stent at 18 atm for 10 seconds at the level of left anterior descending artery obtaining a satisfactory angiographic result. 


\section{Procedure}

Through a conventional right femoral approach, the left coronary ostium was canalized using a $4.06 \mathrm{Fr}$ Launcher guiding catheter (Medtronic). Subsequently, a floppy micro guide at the level of the anterior left descending artery was used to cross the lesion. Balloon dilation was performed to open the vessel starting with a $1.5 \times 18 \mathrm{~mm}$ Sprinter balloon (Medtronic) followed by a $2.0 \times 22 \mathrm{~mm}$ to finally deploy a $2.5 \times 18 \mathrm{~mm}$ Endeavorstent at $18 \mathrm{~atm}$ for 10 seconds at the level of left anterior descending artery obtaining a satisfactory angiographic result (Figure 3-6).

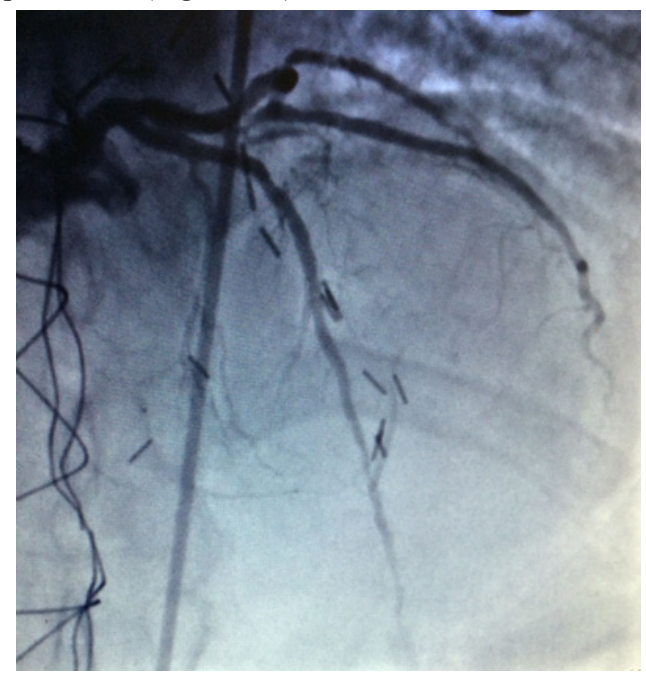

Figure 4 Through a conventional right femoral approach, the left coronary ostium was canalized using a 4.0 6Fr Launcher guiding catheter (Medtronic). Subsequently, a floppy microguide at the level of the anterior left descending artery was used to cross the lesion. Balloon dilation was performed to open the vessel starting with a $1.5 \times 18 \mathrm{~mm}$ Sprinter balloon (Medtronic) followed by a $2.0 \times 22 \mathrm{~mm}$ to finally deploy a $2.5 \times 18 \mathrm{~mm}$ Endeavor stent at $18 \mathrm{~atm}$ for 10 seconds at the level of left anterior descending artery obtaining a satisfactory angiographic result.

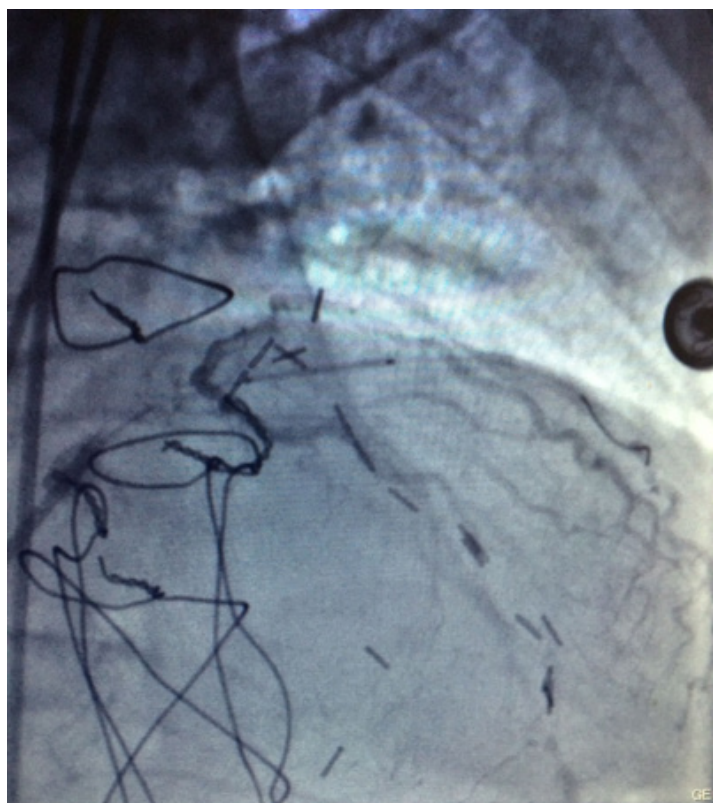

Figure 5 Five months later we decided to treat the ramus intermedius. We predilated the lesion with a $2.5 \times 22 \mathrm{~mm}$ Sprinter Balloon (Medtronic) to place a $2.5 \times 22 \mathrm{~mm}$ Endeavor stent inflated at $16 \mathrm{~atm}$ for 10 seconds obtaining good angiographic result.

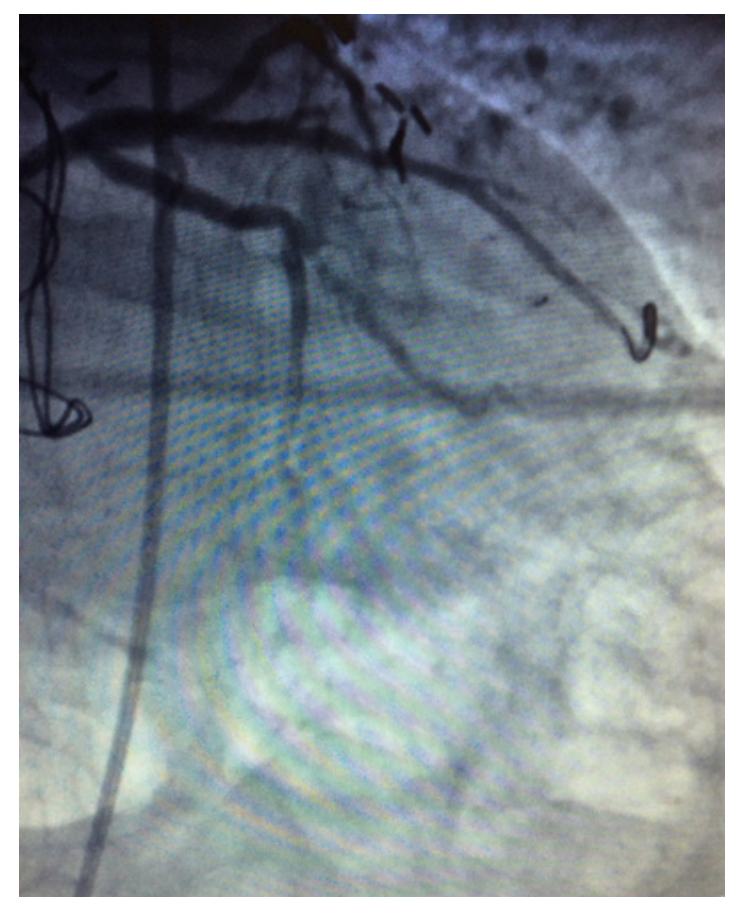

Figure 6 Five months later we decided to treat the ramus intermedius. We predilated the lesion with a $2.5 \times 22 \mathrm{~mm}$ Sprinter Balloon (Medtronic) to place a $2.5 \times 22 \mathrm{~mm}$ Endeavor stent inflated at 16 atm for 10 seconds obtaining good angiographic result.

Five months later we decided to treat the ramus intermedius. We predilated the lesion with a $2.5 \times 22 \mathrm{~mm}$ Sprinter Balloon (Medtronic) to place a $2.5 \times 22 \mathrm{~mm}$ Endeavor stent inflated at 16 atm for 10 seconds obtaining good angiographic result.

\section{Conclusion}

The patient is currently asymptomatic living a normal life. Stress tests were both clinically and electrically negative for myocardial ischemia with good physical capacity.

\section{Acknowledgments}

None.

\section{Conflicts of interest}

Author declares there are no conflicts of interest.

\section{Funding}

None. 